\title{
ATOMIC POLYMORPHISM AND THE EXISTENCE PROPERTY
}

\author{
GILDA FERREIRA
}

\begin{abstract}
We present a purely proof-theoretic proof of the existence property for the full intuitionistic first-order predicate calculus, via natural deduction, in which commuting conversions are not needed. Such proof illustrates the potential of an atomic polymorphic system with only three generators of formulas - conditional and first and second-order universal quantifiers - as a tool for proof-theoretical studies.
\end{abstract}

\section{INTRODUCTION}

In [5] it was shown that there is a purely proof-theoretic proof of the disjunction property for the full intuitionistic propositional calculus (IPC), via natural deduction without the need of commuting conversions. Such a proof relies in a sound and faithful embedding of IPC into an atomic polymorphic system with only two generators of formulas - conditional and second-order universal quantifier - denoted by $\mathbf{F}_{\text {at }}$.

Is it possible to give an alternative proof of the existence property for the full intuitionistic first-order predicate calculus (IQC) in the above commuting conversionfree manner? We show in this paper that the answer is yes. The atomic polymorphic system used to interpret $\mathbf{I Q C}$, which we denote by $\mathbf{F}_{\mathbf{a t}}^{\mathbf{Q}}$, was introduced in [3] under the designation of atomic QSOL ${ }^{i}$. Such system, which we review in the section below, has three generators of formulas, the conditional and the first and second-order universal quantifiers.

Using atomic polymorphic systems to avoid complications of the natural deduction calculus is not a novelty. The defects of the latter calculus were eloquently exposed in [8]. According to Jean-Yves Girard et al (page 74) 'The elimination rules [for $\perp, \vee$ and $\exists$ ] are very bad.' and proceed 'Indeed, we cannot decently work with the full fragment without identifying a priori different deduction... the need to add these supplementary rules [referring to the commuting conversions for $\perp$, $\checkmark$ and $\exists$ ] reveals an inadequacy of the syntax.' Fernando Ferreira coined a simple and elegant way of avoiding the 'inadequacy' of the natural deduction calculus in the context of IPC presenting in [2] an embedding of IPC into $\mathbf{F}_{\mathbf{a t}}{ }^{1}$ - a predicative system without bad connectives. Several studies in IPC taking advantage of its embedding into $\mathbf{F}_{\text {at }}$ followed. See for example [4, 5, 6, 7]. F. Ferreira and

2010 Mathematics Subject Classification. 03F07; 03F03; 03B20; 03F05.

Key words and phrases. predicative polymorphism, intuitionistic predicate calculus, existence property, natural deduction, normalization, faithfulness.

The author acknowledges the support of Fundação para a Ciência e a Tecnologia [UID/MAT/04561/2013, UID/CEC/00408/2013 and grant SFRH/BPD/93278/2013] and is also grateful to Centro de Matemática, Aplicações Fundamentais e Investigação Operacional and LargeScale Informatics Systems Laboratory.

${ }^{1}$ In paper [2] $\mathbf{F}_{\text {at }}$ was designated by atomic PSOL ${ }^{\mathrm{i}}$. 
the author showed in [3] that the previous embedding could be extended to the full intuitionistic first-order predicate calculus. More precisely, it is possible to embed IQC into an atomic polymorphic calculus $\left(\mathbf{F}_{\mathbf{a t}}^{\mathbf{Q}}\right)$ with implication and first and second-order universal quantifiers, i.e., still without bad connectives. In the present paper, having in view to show that the above embedding into $\mathbf{F}_{\mathbf{a t}}^{\mathbf{Q}}$ can be a useful tool for IQC, we prove the faithfulness of such embedding and explore some proof-theoretic properties of $\mathbf{F}_{\mathbf{a t}}^{\mathbf{Q}}$. Namely, we show that if the translation of $\exists x . A$ is derivable in $\mathbf{F}_{\mathbf{a t}}^{\mathbf{Q}}$ then there is a first-order term $t$ such that the translation of $A[t / x]$ is derivable in $\mathbf{F}_{\mathbf{a t}}^{\mathbf{Q}}$. Note that in order to obtain $t$ we do not rely on commuting conversions. As an illustration of the embedding's potential we show that the existence property for IQC can be easily derived from the homologous property in the atomic polymorphic context. Not surprisingly, in this alternative proof of the existence property for IQC commuting conversions are absent.

The paper is organized as follows: in Section 2 we review the atomic polymorphic system $\mathbf{F}_{\mathbf{a t}}^{\mathbf{Q}}$ and the sound translation of $\mathbf{I Q C}$ into $\mathbf{F}_{\mathbf{a t}}^{\mathbf{Q}}$ and we prove some properties used in following sections such as strong normalization and faithfulness for $\mathbf{F}_{\mathbf{a t}}^{\mathbf{Q}}$; in Section 3, we prove that $\mathbf{F}_{\mathbf{a}}^{\mathbf{Q}}$ enjoys the existence property and in Section 4 we present an alternative proof of the existence property for IQC, via natural deduction, without commuting conversions.

\section{ATOMIC POLYMORPHISM FOR IQC}

The atomic polymorphic calculus used in this paper is $\mathbf{F}_{\mathbf{a t}}^{\mathbf{Q}}$, a calculus described in $[3]^{2}$, which has first and second-order constants, first-order variables $x, y, z$, $\ldots$, sentential variables $X, Y, Z, \ldots$ and the connectives: $\rightarrow$ (conditional), $\forall_{1}$ (first-order universal quantifier) and $\forall_{2}$ (second-order universal quantifier). Atomic formulas are either second-order constants and variables or expressions of the form $P\left(t_{1}, \ldots, t_{n}\right)$, where $P$ is a $n$-ary relational symbol and $t_{1}, \ldots, t_{n}$ are first-order terms. The class of formulas is built in the usual way from atomic formulas, closing the class under $\rightarrow, \forall_{1}$ and $\forall_{2}$. I.e. if $A$ and $B$ are formulas, $x$ is a first-order variable and $X$ is a second-order variable then $A \rightarrow B, \forall_{1} x$. $A$ and $\forall_{2} X$. $A$ are also formulas. The subscripts in $\forall_{1}$ and $\forall_{2}$ are usually omitted. In what follows, the language of $\mathbf{F}_{\text {at }}^{\mathbf{Q}}$ will be denoted by $\mathcal{L}$.

The logic of $\mathbf{F}_{\mathbf{a t}}^{\mathbf{Q}}$ is intuitionistic logic that we formulate in the natural deduction calculus through the usual introduction and elimination rules for $\rightarrow, \forall_{1}$ and $\forall_{2}$.

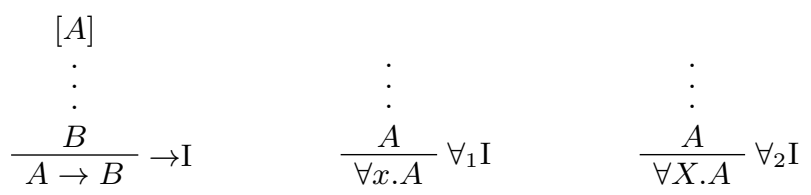

where $x$ and $X$ do not occur free in any undischarged hypothesis (respectively),

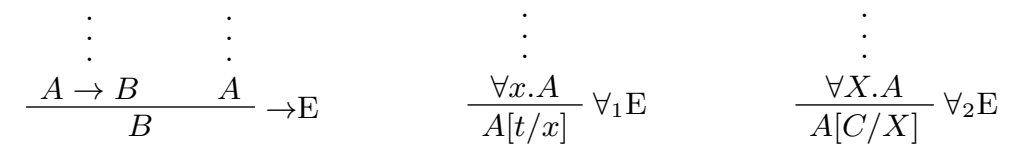

${ }^{2}$ In [3], system $\mathbf{F}_{\mathbf{a t}}^{\mathbf{Q}}$ was denoted by atomic QSOL ${ }^{\mathrm{i}}$. 
with $t$ a term (free for $x$ in $A$ ), $C$ an atomic formula (free for $X$ in $A$ ), and $A[\beta / \alpha]$ results from $A$ by replacing all the free occurrences of $\alpha$ by $\beta$.

Note the restriction to atomic instantiations in the $\forall_{2}$ E-rule of $\mathbf{F}_{\mathbf{a t}}^{\mathbf{Q}}$ as opposed to the well-known Girard/Reynold's impredicative polymorphic system $\mathbf{F}[8,10]$ which allows instantiation with any (not necessarily atomic) formula. The severe restriction above reduces enormously the expressive power of the system but has the advantage of keeping it predicative and with a natural notion of subformula the immediate subformulas of the formula $\forall X$. $A$ are the formulas $A[C / X]$ with $C$ an atomic formula (free for $X$ in $A$ ).

Furthermore, as shown in [3], $\mathbf{F}_{\mathbf{a t}}^{\mathbf{Q}}$ is expressive enough to interpret the (full) intuitionistic first-order predicate calculus. I.e. via the following Russell-Prawitz translation $([9,11])$ of formulas from $\mathbf{I Q C}$ into $\mathbf{F}_{\mathbf{a t}}^{\mathbf{Q}}$

$$
\begin{aligned}
& \left(P\left(t_{1}, \ldots, t_{n}\right)\right)^{*}: \equiv P\left(t_{1}, \ldots, t_{n}\right), \text { with } P \text { a relational symbol and } t_{i} \text { terms } \\
& (\perp)^{*}: \equiv \forall X . X \\
& (A \rightarrow B)^{*}: \equiv A^{*} \rightarrow B^{*} \\
& (A \wedge B)^{*}: \equiv \forall X .\left(\left(A^{*} \rightarrow\left(B^{*} \rightarrow X\right)\right) \rightarrow X\right) \\
& (A \vee B)^{*}: \equiv \forall X .\left(\left(A^{*} \rightarrow X\right) \rightarrow\left(\left(B^{*} \rightarrow X\right) \rightarrow X\right)\right) \\
& (\forall x . A)^{*}: \equiv \forall x . A^{*} \\
& (\exists x . A)^{*}: \equiv \forall X .\left(\forall x .\left(A^{*} \rightarrow X\right) \rightarrow X\right),
\end{aligned}
$$

where $X$ is a second-order variable which does not occur in $A^{*}$ nor in $B^{*}$, we have that

$$
\text { if } \Gamma \vdash_{\mathbf{I Q C}} A \text { then } \Gamma^{*} \vdash_{\mathbf{F}_{\mathbf{a t}}^{\mathbf{Q}}} A^{*},
$$

where $\Gamma: \equiv A_{1}, \ldots, A_{n}$ and $A_{1}, \ldots, A_{n}, A$ are formulas in $\mathbf{I Q C}$ and $\Gamma^{*}: \equiv A_{1}^{*}, \ldots, A_{n}^{*}$.

The soundness proof relies on the phenomenon of instantiation overflow. Instantiation overflow ensures that, from formulas of the form

$$
\begin{aligned}
& \forall X . X \\
& \forall X .((A \rightarrow(B \rightarrow X)) \rightarrow X) \\
& \forall X .((A \rightarrow X) \rightarrow((B \rightarrow X) \rightarrow X)) \\
& \forall X .(\forall x .(A \rightarrow X) \rightarrow X),
\end{aligned}
$$

it is possible to deduce in $\mathbf{F}_{\mathbf{a t}}^{\mathbf{Q}}$ (respectively)

$$
\begin{aligned}
& F \\
& (A \rightarrow(B \rightarrow F)) \rightarrow F \\
& A \rightarrow F) \rightarrow((B \rightarrow F) \rightarrow F) \\
& \forall x .(A \rightarrow F) \rightarrow F,
\end{aligned}
$$

for any (not necessarily atomic) formula $F$. See [3] for a detailed proof including the algorithmic methods for instantiation overflow. For more information on instantiation overflow see also [1].

The result below follows easily from the Russell-Prawitz translation:

Lemma 2.1. Let $A$ be a formula in $\mathbf{I Q C}$ and $A^{*}$ its translation into $\mathbf{F}_{\mathbf{a t}}^{\mathbf{Q}}$. Then

i) $A^{*}$ has no second-order free variables.

ii) Second-order universal quantifications in $A^{*}$ always occur in one of the following four specific forms:

a) $\forall X . X$,

b) $\forall X .\left(\left(D^{*} \rightarrow\left(E^{*} \rightarrow X\right)\right) \rightarrow X\right)$,

c) $\forall X .\left(\left(D^{*} \rightarrow X\right) \rightarrow\left(\left(E^{*} \rightarrow X\right) \rightarrow X\right)\right)$ or

d) $\forall X .\left(\forall x .\left(D^{*} \rightarrow X\right) \rightarrow X\right)$ 
with $D$ and $E$ formulas in $\mathbf{I Q C}$. The formulas in $a), b), c)$ and $d$ ) are the translations into $\mathbf{F}_{\mathbf{a t}}^{\mathbf{Q}}$ of the subformulas of $A: \perp, D \wedge E, D \vee E$ and $\exists x . D$ respectively.

A normalization theorem for $\mathbf{F}_{\mathbf{a t}}^{\mathbf{Q}}$ can be easily proved in the manner of Theorem 2 of section $\mathrm{V}$ of [9]. And normalization for $\mathbf{F}_{\mathbf{a t}}^{\mathbf{Q}}$ (considering $\beta$-conversions) is enough for the purposes of the present paper. However, since the strategy to prove strong normalization for $\mathbf{F}_{\text {at }}$ presented in [4] can be adapted to prove strong normalization for $\mathbf{F}_{\mathbf{a t}}^{\mathbf{Q}}$, we sketch here such adaptation showing that $\mathbf{F}_{\mathbf{a t}}^{\mathbf{Q}}$ enjoys the strong normalization property considering $\beta \eta$-conversions.

By the Curry-Howard isomorphism also known as "formulas-as-types paradigm", $\mathbf{F}_{\mathbf{a t}}^{\mathbf{Q}}$ can be presented in the (operational) $\lambda$-calculus style. Types in $\mathbf{F}_{\mathbf{a t}}^{\mathbf{Q}}$ are constructed from atomic types (second-order constants, second-order variables and expressions of the form $P\left(t_{1}, \ldots, t_{n}\right)$, with $P$ a relational symbol and $t_{i}$ first-order terms) by means of three type-forming operations $\rightarrow, \forall_{1}$ and $\forall_{2}$. I.e. atomic types are types and if $A$ and $B$ are types, $x$ is a first-order variable and $X$ is a secondorder variable then $A \rightarrow B, \forall x . A, \forall X . A$ are types. In $\mathbf{F}_{\mathrm{at}}^{\mathbf{Q}}, \lambda$-terms are generated by the following clauses:

(i) For each type $A$ there are countably infinite many assumption variables of type $A, x^{A}, y^{A}, z^{A}$, etc. Assumption variables are $\lambda$-terms.

(ii) If $t^{A \rightarrow B}$ and $q^{A}$ are $\lambda$-terms of types $A \rightarrow B$ and $A$, respectively, then $\left(t^{A \rightarrow B} q^{A}\right)^{B}$ is a $\lambda$-term of type $B$.

(iii) If $t^{B}$ is a $\lambda$-term of type $B$ and $x^{A}$ is an assumption variable of type $A$, then $\left(\lambda x^{A} \cdot t^{B}\right)^{A \rightarrow B}$ is a $\lambda$-term of type $A \rightarrow B$. A $\lambda$-term of this form is called an arrow abstraction.

(iv) If $t^{\forall X . A}$ is a $\lambda$-term of type $\forall X . A$ and $C$ in an atomic type, then $\left(t^{\forall X . A} C\right)^{A[C / X]}$ is a $\lambda$-term of type $A[C / X]^{3}$.

(v) If $t^{A}$ is a $\lambda$-term of type $A$ and the type variable $X$ is not free in the type of any free assumption variable of $t^{A}$, then $\left(\Lambda X . t^{A}\right)^{\forall X . A}$ is a $\lambda$-term of type $\forall X$.A. A $\lambda$-term of this form is called a second-order universal abstraction.

(vi) If $t^{\forall x . A}$ is a $\lambda$-term of type $\forall x . A$ and $q$ is a first-order term of $\mathcal{L}$ then $\left(t^{\forall x \cdot A} q\right)^{A[q / x]}$ is a $\lambda$-term of type $A[q / x]$.

(vii) If $t^{A}$ is a $\lambda$-term of type $A$ and the first-order variable $x$ is not free in the type of any free assumption variable of $t^{A}$, then $\left(\Lambda_{1} x \cdot t^{A}\right)^{\forall x . A}$ is a $\lambda$ term of type $\forall x$.A. A $\lambda$-term of this form is called a first-order universal abstraction.

Note that (although sometimes represented by the same letters) first-order variables and assumption variables are distinct entities. Assumption variables are $\lambda$ terms and always have a type associated. Assumption variables never occur in types. First-order variables are variables of the language $\mathcal{L}$ and may occur in $\lambda$ terms, inside or outside its type. First-order variables never have a type associated. For example, in the $\lambda$-term $\left(y^{\forall x A} z\right)^{A[z / x]}$ of type $A[z / x], y$ is an assumption variable of type $\forall x A$ and $x$ and $z$ are first-order variables. A comment similar to the previous one may be made apropos first-order terms of $\mathcal{L}$ and $\lambda$-terms.

\footnotetext{
${ }^{3}$ By regarding types as formulas, we have the usual definitions of free and bound variables in a type. We denote the set of free variables in $A$ by $\mathrm{FV}(A)$. We write $A[\beta / \alpha]$ for the type obtained from $A$ by substituting the free occurrences of $\alpha$ in $A$ by $\beta$. We assume w.l.o.g. that $\beta$ is free for $\alpha$ in $A$. As usual, we can freely rename the bound variables in a type.
} 
In the inductive definition of the free variables of a $\lambda$-term (definition 3 of [4], page 262), we have now two new clauses: $\mathrm{FV}\left(\Lambda_{1} x . t\right): \equiv \mathrm{FV}(t) \backslash\{x\}$ and $\mathrm{FV}(t q): \equiv$ $\mathrm{FV}(t) \cup \mathrm{V}(q)$ with $t$ a $\lambda$-term, $q$ a first-order term and $\mathrm{V}(q)$ the set of variables in $q$. In the replacement of a second-order free variable $X$ in a $\lambda$-term $t$ by an atomic type $C$, denoted by $t[C / X]$, according to [4] definition 4 page 262, we have two new cases: $\left(\Lambda_{1} x . s\right)[C / X]: \equiv \Lambda_{1} x .(s[C / X])$ and $(s q)[C / X]: \equiv s[C / X] q$, with $s$ a $\lambda$-term and $q$ a first-order term. The substitution of a free assumption variable $x^{A}$ in a $\lambda$-term $t$ by a $\lambda$-term $s^{A}$ or the substitution of a first-order free variable $y$ in a $\lambda$-term $t$ by a first-order term $q$ are done in the expected way and are denoted by $t\left[s^{A} / x^{A}\right]$ and $t[q / y]$ respectively. For example $\left(\Lambda_{1} x . t\right)[q / x]: \equiv \Lambda_{1} x . t ;\left(\Lambda_{1} x . t\right)[q / y]: \equiv \Lambda_{1} x .(t[q / y])$, for $x \not \equiv y$ and $(t q)\left[s^{A} / x^{A}\right]: \equiv t\left[s^{A} / x^{A}\right] q$, for $q$ a first-order term.

Note that in $\mathbf{F}_{\text {at }}^{\mathbf{Q}}$ we have the same conversions we have in $\mathbf{F}_{\text {at }}$ plus the following two ( $\beta$ and $\eta$ respectively) conversions for $\Lambda_{1}$ :

$$
\begin{array}{lll}
\left(\Lambda_{1} x . t\right) q & \rightsquigarrow t[q / x] \\
\Lambda_{1} x .(t x) & \rightsquigarrow t, \quad \text { with } x \notin \mathrm{FV}(t)
\end{array}
$$

Remember that the strategy in [4] to prove that $\mathbf{F}_{\text {at }}$ enjoys the strong normalization property (a simple adaptation of Tait's convertibility technique) proceeds as follows: i) we define by induction on the complexity of the types a class Red of $\lambda$-terms of $\mathbf{F}_{\text {at }}$; ii) we prove that all $\lambda$-terms in Red are strongly normalizable considering $\beta \eta$-conversions; iii) we prove that all $\lambda$-terms in $\mathbf{F}_{\text {at }}$ are in Red.

Remember also that Red is defined in the following way:

For $C$ an atomic type, $t \in \operatorname{Red}_{C}: \equiv t$ is strongly normalizable. $t \in \operatorname{Red}_{A \rightarrow B}: \equiv$ for all $\lambda$-term $s$, if $s \in \operatorname{Red}_{A}$ then $t s \in \operatorname{Red}_{B}$.

$t \in \operatorname{Red}_{\forall X . A}: \equiv$ for all atomic types $C, t C \in \operatorname{Red}_{A[C / X]}$.

In the context of $\mathbf{F}_{\mathbf{a t}}^{\mathbf{Q}}$ we only have to add a new clause for first-order universal quantification:

$$
t \in \operatorname{Red}_{\forall x . A}: \equiv \text { for all first-order term } s \text { in } \mathcal{L}, t s \in \operatorname{Red}_{A[s / x]} .
$$

We say that a $\lambda$-term is reducible if it belongs to Red and we say that a $\lambda$-term is neutral if it is not of the form $\lambda$ x.t, $\Lambda_{1}$ x.t or $\Lambda$ X.t, i.e., it is not an abstraction.

The proof that every $\lambda$-term in Red is strongly normalizable follows from the following lemma (where $t \succeq q$ means that $t \beta \eta$-reduces to $q$ and $t \succ_{1} q$ means that $t \beta \eta$-reduces to $q$ in one step):

Lemma 2.2. $\operatorname{Red}_{A}$ satisfies the following three conditions:

(CR 1) If $t \in \operatorname{Red}_{A}$ then $t$ is strongly normalizable.

(CR 2) If $t \in \operatorname{Red}_{A}$ and $t \succeq t^{\prime}$ then $t^{\prime} \in \operatorname{Red}_{A}$.

(CR 3) If $t$ is neutral and $t^{\prime} \in \operatorname{Red}_{A}$ for all $t^{\prime}$ such that $t \succ_{1} t^{\prime}$, then $t \in \operatorname{Red}_{A}$.

As a special case of CR 3, we have in particular:

(CR 4) If $t^{A}$ is neutral and normal then $t \in \operatorname{Red}_{A}$.

Proof. The proof is by induction on the complexity of the type A. For the atomic, arrow and second-order universal types, the proof follows exactly as in [4], lemma 1. Let us analyze the type $A: \equiv \forall x . B$.

Take $t \in \operatorname{Red}_{\forall x . B}$ in order to prove that $t$ is strongly normalizable. By definition of $\operatorname{Red}_{\forall x . B}, t x \in \operatorname{Red}_{B}$. By induction hypothesis, $\operatorname{Red}_{B}$ satisfies CR 1, so $t x$ is strongly normalizable. Clearly, each reduction sequence for $t$ gives rise to a reduction sequence for $t x$ by applying each term of the sequence to $x$. Hence, $t$ is strongly normalizable. 
In order to prove CR 2 , let $t \in \operatorname{Red}_{\forall x . B}$ and $t \succeq t^{\prime}$. We want to show that $t^{\prime} \in \operatorname{Red}_{\forall x . B}$. Let $q$ be a first-order term of $\mathcal{L}$. We know that $t q \in \operatorname{Red}_{B[q / x]}$. Since $t q \succeq t^{\prime} q$, by induction hypothesis (CR 2, for $\left.\operatorname{Red}_{B[q / x]}\right)$, we have $t^{\prime} q \in \operatorname{Red}_{B[q / x]}$.

For condition CR 3, take $t^{\forall x . B}$ as in the hypothesis. We want to prove that $t \in \operatorname{Red}_{\forall x . B}$. Let $q$ be a first-order term of $\mathcal{L}$. Note that $t q$ is neutral and that the redexes in $t q$ occur in $t$ (since $t$ is neutral and, hence, not a first-order universal abstraction). Therefore, any single reduction step from $t q$ has the form $t^{\prime} q$ with $t \succ_{1} t^{\prime}$. By hypothesis $t^{\prime} \in \operatorname{Red}_{\forall x . B}$, and we get $t^{\prime} q \in \operatorname{Red}_{B[q / x]}$. Applying now the induction hypothesis $\left(\operatorname{CR} 3\right.$ for $\left.\operatorname{Red}_{B[q / x]}\right)$, we conclude that $t q \in \operatorname{Red}_{B[q / x]}$.

The proof that all $\lambda$-terms of $\mathbf{F}_{\text {at }}^{\mathbf{Q}}$ are in Red is immediate from Proposition 2.4 below ${ }^{4}$. First we need an auxiliary lemma:

Lemma 2.3. In $\mathbf{F}_{\text {at }}^{\mathbf{Q}}$ we have the following:

(i) If for all reducible $r$ of type $A, t\left[r / x^{A}\right]$ is reducible, then so is $\lambda x . t$.

(ii) If for all atomic types $C, t[C / X]$ is reducible and $X$ is not free in the type of a free assumption variable of $t$, then $\Lambda X$.t is reducible.

(iii) If for all first-order terms $s$ in $\mathcal{L}, t[s / x]$ is reducible and $x$ is not free in the type of a free assumption variable of $t$, then $\Lambda_{1} x . t$ is reducible.

Proof. We prove (iii). Assertions (i) and (ii) are proved in a similar way and such proofs, in the context of $\mathbf{F}_{\text {at }}$, can be found in [4], page 265 .

Let us prove that, for all $\lambda$-terms $t$ satisfying the conditions of Lemma 2.3 (iii), we have: For all first-order term $q,\left(\Lambda_{1} x . t\right) q$ is reducible. The proof is by induction on $\mu(t)$, i.e, on a number that bounds the length of every reduction sequence beginning with $t$. (Indeed, $t$ is strongly normalizable because $t$ is $t[x / x]$ which, by supposition, is reducible and hence, by $\mathrm{CR} 1$ is strongly normalizable.)

Fix $q$ a first-order term. Since the $\lambda$-term $\left(\Lambda_{1} x . t\right) q$ is neutral, we only have to show that the one-step reducts from this term are reducible (thanks to CR 3 ). Such a one step reduct must be of the form $t[q / x],\left(\Lambda_{1} x \cdot t^{\prime}\right) q$ with $t \succ_{1} t^{\prime}$ or $s q$, when $t$ is $s x$ and $x$ does not occur free in $s$. In the first case, reducibility follows from the hypothesis. In the second case, note that $\mu\left(t^{\prime}\right)<\mu(t)$ and that, for all first-order terms $q, t^{\prime}[q / x]$ is reducible (the last assertion uses CR 2). So, by induction hypothesis, $\left(\Lambda_{1} x . t^{\prime}\right) q$ is reducible. The third case reduces to the first since $s q \equiv(s x)[q / x] \equiv t[q / x]$.

Proposition 2.4. Let $t$ be any $\lambda$-term (not assumed to be reducible) and suppose that all the free assumption variables of $t$ are among $x_{1}, \ldots, x_{n}$, of types $A_{1}, \ldots, A_{n}$ (respectively). Suppose, in addition, that all the first-order free variables of $t$ are among $y_{1}, \ldots, y_{r}$ and all the second-order free variables of $t$ are among $X_{1}, \ldots, X_{m}$. If $q_{1}, \ldots, q_{r}$ are terms of $\mathcal{L}, C_{1}, \ldots, C_{m}$ are atomic types and $s_{1}, \ldots, s_{n}$ are reducible $\lambda$-terms of types $A_{1}[\bar{C} / \bar{X}][\bar{q} / \bar{y}], \ldots, A_{n}[\bar{C} / \bar{X}][\bar{q} / \bar{y}]$ then the term $t[\bar{C} / \bar{X}][\bar{q} / \bar{y}][\bar{s} / \bar{x}]$ is reducible.

Proof. The proof of the result above is done by induction on the complexity of the $\lambda$-term $t$. We illustrate with the two new $\lambda$-terms and with the $\lambda$-term $t: \equiv \Lambda Y$.p. All the other cases are easy adaptations of the proof of proposition 1 in [4].

If $t: \equiv\left(p^{\forall z \cdot B} u\right)^{B[u / z]}$, with $u$ a term of $\mathcal{L}$, we want to prove that the $\lambda$ term $(p u)[\bar{C} / \bar{X}][\bar{q} / \bar{y}][\bar{s} / \bar{x}]$ with $\bar{q}$ terms of $\mathcal{L}, \bar{C}$ atomic types and $\bar{s}$ reducible

\footnotetext{
${ }^{4}$ In such proposition just take $q_{i}$ as being $y_{i}, C_{i}$ as being $X_{i}$ and $s_{i}$ as being $x_{i}$. Note that, by $\mathrm{CR} 4$, an assumption variable is a reducible $\lambda$-term.
} 
$\lambda$-terms of type $\bar{A}[\bar{C} / \bar{X}][\bar{q} / \bar{y}]$, is reducible. By induction hypothesis we know that $p[\bar{C} / \bar{X}][\bar{q} / \bar{y}][\bar{s} / \bar{x}]$ is reducible of type $\forall z \cdot(B[\bar{C} / \bar{X}][\bar{q} / \bar{y}])$. By definition of reducibility we infer that the $\lambda$-term $(p[\bar{C} / \bar{X}][\bar{q} / \bar{y}][\bar{s} / \bar{x}]) u[\bar{q} / \bar{y}]$ is reducible of type $B[\bar{C} / \bar{X}][\bar{q} / \bar{y}][u[\bar{q} / \bar{y}] / z]$. Just note that the latter $\lambda$-term is $(p u)[\bar{C} / \bar{X}][\bar{q} / \bar{y}][\bar{s} / \bar{x}]$.

If $t: \equiv \Lambda_{1} z \cdot p$, we want to prove that $\left(\Lambda_{1} z \cdot p\right)[\bar{C} / \bar{X}][\bar{q} / \bar{y}][\bar{s} / \bar{x}]$ is reducible. Note that, by the freely renaming of bound variables, we may assume w.l.o.g. that $z$ is a new variable, not occurring in $\bar{C}, \bar{q}$, nor in $\bar{s}$. By definition of substitution, the $\lambda$-term above is $\Lambda_{1} z \cdot(p[\bar{C} / \bar{X}][\bar{q} / \bar{y}][\bar{s} / \bar{x}])$. By Lemma 2.3 (iii), it is enough to show that for all terms $v$ of $\mathcal{L}, p[\bar{C} / \bar{X}][\bar{q} / \bar{y}][\bar{s} / \bar{x}][v / z]$ is reducible. But this latter $\lambda$-term is $p[\bar{C} / \bar{X}][\bar{q} / \bar{y}, v / z][\bar{s} / \bar{x}]$ which is reducible by induction hypothesis.

If $t: \equiv \Lambda Y \cdot p$, we want to prove that $(\Lambda Y \cdot p)[\bar{C} / \bar{X}][\bar{q} / \bar{y}][\bar{s} / \bar{x}] \equiv \Lambda Y \cdot(p[\bar{C} / \bar{X}][\bar{q} / \bar{y}][\bar{s} / \bar{x}])$ is reducible. Following the proof in [4] page 266, we know that it is enough to prove that for all atomic type $D,(p[\bar{C} / \bar{X}][\bar{q} / \bar{y}][\bar{s} / \bar{x}])[D / Y]$ is reducible. Note that, taking $\bar{w}$ a $r$-tuple of new first-order variables we have $(p[\bar{C} / \bar{X}][\bar{q} / \bar{y}][\bar{s} / \bar{x}])[D / Y] \equiv$ $(p[\bar{w} / \bar{y}][\bar{C}[\bar{q} / \bar{y}] / \bar{X}][\bar{q} / \bar{w}][\bar{s} / \bar{x}])[D / Y] \equiv(p[\bar{w} / \bar{y}][\bar{C}[\bar{q} / \bar{y}] / \bar{X}][D / Y][\bar{q} / \bar{w}][\bar{s} / \bar{x}])$. The result follows applying the induction hypothesis to $p[\bar{w} / \bar{y}]$. Note that $\bar{C}[\bar{q} / \bar{y}]$ are still atomic types and $\bar{s}$ are reducible terms of type $\bar{A}[\bar{C} / \bar{X}][\bar{q} / \bar{y}] \equiv \bar{A}[\bar{w} / \bar{y}][\bar{C}[\bar{q} / \bar{y}] / \bar{X}][\bar{q} / \bar{w}]$.

After this brief detour to show that system $\mathbf{F}_{\mathbf{a t}}^{\mathbf{Q}}$ is strongly normalizable, we return to natural deduction notation and we prove that $\mathbf{F}_{\mathbf{a t}}^{\mathbf{Q}}$ enjoys the following stronger version of the subformula property (first presented in [8] page 76 in the fragment $\left.\left(\wedge, \rightarrow, \forall_{1}\right)\right)$ :

Proposition 2.1. Let $\Delta$ be a normal derivation in $\mathbf{F}_{\mathbf{a t}}^{\mathbf{Q}}$. Then

i) Every formula in $\Delta$ is a subformula of the conclusion or of a (undischarged) hypothesis of $\Delta$. [Subformula Property]

ii) If $\Delta$ ends in an elimination rule, it has a principal branch, i.e. a sequence of formulas $A_{0}, A_{1}, \ldots, A_{n}$ such that

- $A_{0}$ is an (undischarged) hypothesis;

- $A_{n}$ is the conclusion;

- for $i=0, \ldots, n-1, A_{i}$ is the principal premise (i.e. the premise that carries the eliminated symbol) of an elimination rule whose conclusion is $A_{i+1}$.

In particular, $A_{n}$ is a subformula of $A_{0}$.

Proof. The proof is done by induction on the number of inferences in $\Delta$ as in [8]. The rules for second-order universal quantification can be dealt with exactly in the same way and were analysed in [2].

By a translated formula in $\mathbf{F}_{\mathbf{a t}}^{\mathbf{Q}}$ we mean a formula in $\mathbf{F}_{\mathbf{a t}}^{\mathbf{Q}}$ which is the translation (via the Russell-Prawitz translation) of a certain formula in IQC.

Lemma 2.5. Let $\mathcal{D}$ be a derivation in $\mathbf{F}_{\mathbf{a t}}^{\mathbf{Q}}$ of a formula $A$ from assumptions $\Gamma$. Let $X$ be a second-order variable and $F$ be a formula in $\mathbf{F}_{\mathbf{a t}}^{\mathbf{Q}}$. If all second-order universal formulas in $\mathcal{D}$ and in $\Gamma$ are subformulas of translated formulas, then $\mathcal{D}$ can be transformed into a (normal) derivation of $A[F / X]$ from $\Gamma[F / X]$.

Proof. The proof is by induction on the number of inferences in $\mathcal{D}$. If $\mathcal{D}$ consists solely of an hypothesis (i.e. $A$ is derived from $A$ ), the result is immediate: $A[F / X]$ is derived from $A[F / X]$. 
For the implication rules, for the first-order universal rules, for the $\forall_{2} \mathrm{I}$-rule and for the $\forall_{2}$ E-rule when the conclusion is not obtained by instantiating the secondorder bound variable of the universal quantifier by the atomic variable $X$ the result follows by induction hypothesis. Just notice that the rules are still valid when replacing the free occurrences of $X$ by $F$. We are assuming w.l.o.g. (modulo a renaming of variables) that the first and second-order bounded variables in $\mathcal{D}$ do not occur free in $F$ and are not $X$.

Let us analyse a $\forall_{2}$ E-rule of the form $\frac{\forall Y . H}{H[X / Y]}$. By induction hypothesis we have that from $\Gamma[F / X]$ we can derive (in $\left.\mathbf{F}_{\mathbf{a t}}^{\mathbf{Q}}\right)(\forall Y . H)[F / X]$. By hypothesis we know that all the second-order universal formulas in $\mathcal{D}$ (and in particular $\forall Y . H$ ) are subformulas of translated formulas. From Proposition 2.1, easily we conclude that $\forall Y . H$ is of one of the four logical types listed in Proposition 2.1-ii) which enjoy the property of instantiation overflow and $\forall Y . H$ has no second-order free variables (thus, $(\forall Y . H)[F / X] \equiv \forall Y . H$ and $H[F / X] \equiv H)$. Therefore, in $\mathbf{F}_{\mathbf{a t}}^{\mathbf{Q}}$, from $\forall Y . H$ we can derive $H[F / Y]$ (instantiation overflow), and thus from $\Gamma[F / X]$ we can derive (in $\left.\mathbf{F}_{\mathbf{a t}}^{\mathbf{Q}}\right) H[F / Y] \equiv H[X / Y][F / X]$.

In an easy adaptation of the faithfulness of $\mathbf{F}_{\text {at }}$ [5] we finish this section proving that the translation of $\mathbf{I Q C}$ into $\mathbf{F}_{\text {at }}^{\mathbf{Q}}$ more than being just sound it is also faithful, i.e., if a translated formula is derivable in this latter calculus, then the original formula is derivable in the predicate calculus.

The proof follows the strategy in [5], pages 1305-1310. It is based on the following (adapted) definition and lemma.

Definition 2.2. Let $A$ be a predicate formula, i.e, a formula in IQC. For $B$ any subformula of $A^{*}$, we define a formula $\tilde{B}$ in the language of predicate calculus $(\perp$, $\wedge, \vee, \rightarrow, \forall_{1}, \exists_{1}$ ) extended with second-order variables (but without second-order quantifications) in the following way:

If $B$ is an atomic formula, then $\tilde{B}: \equiv B$.

If $B: \equiv D \rightarrow E$, then $\tilde{B}: \equiv \tilde{D} \rightarrow \tilde{E}$.

If $B: \equiv \forall x . D$, then $\tilde{B}: \equiv \forall x . \tilde{D}$.

If $B: \equiv \forall X . X$, then $\tilde{B}: \equiv \perp$.

If $B: \equiv \forall X .\left(\left(D^{*} \rightarrow\left(E^{*} \rightarrow X\right)\right) \rightarrow X\right)$, then $\tilde{B}: \equiv D \wedge E$.

If $B: \equiv \forall X .\left(\left(D^{*} \rightarrow X\right) \rightarrow\left(\left(E^{*} \rightarrow X\right) \rightarrow X\right)\right)$, then $\tilde{B}: \equiv D \vee E$.

If $B: \equiv \forall X .\left(\forall x .\left(D^{*} \rightarrow X\right) \rightarrow X\right)$, then $\tilde{B}: \equiv \exists x . D$.

The above definition covers all the subformulas of translated formulas - note that second-order quantifications in a translated formula (see Lemma 2.1) appear in one of the four logical types above. Also note that the target system of the $\widetilde{(\cdot)}$-translation is (not exactly but) almost $\mathbf{I Q C}$ : the formulas of $\mathbf{F}_{\mathbf{a t}}^{\mathbf{Q}}$ that are subformulas of translated formulas are translated via the $\widetilde{(\cdot)}$-interpretation into formulas that are in the language of IQC enriched with second-order variables. And $\widetilde{(\cdot)}$ works as an undo translation of the $(\cdot)^{*}$-translation in the sense that for $D$ a predicate formula, $\widetilde{D^{*}}$ is just $D$.

Lemma 2.6. Let $\Gamma$ be a tuple of formulas in $\mathbf{F}_{\mathbf{a t}}^{\mathbf{Q}}$ and $A$ be a formula in $\mathbf{F}_{\mathbf{a t}}^{\mathbf{Q}}$ with their second-order free variables among the variables in $\bar{X}$. If there is a proof (say $\mathcal{D})$ in $\mathbf{F}_{\mathbf{a t}}^{\mathbf{Q}}$ of $A[\bar{X}]$ from $\Gamma[\bar{X}]$ in which all formulas (occurring in $\mathcal{D}$ and $\Gamma[\bar{X}]$ ) are subformulas of translated formulas, then 


$$
\tilde{\Gamma}[\bar{F} / \bar{X}] \vdash_{\text {IQC }} \tilde{A}[\bar{F} / \bar{X}]
$$

for any tuple of predicate formulas $\bar{F}$. For $\Gamma[\bar{X}]: \equiv A_{1}[\bar{X}], \ldots, A_{n}[\bar{X}], \tilde{\Gamma}[\bar{F} / \bar{X}]$ denotes the tuple of predicate formulas $\tilde{A}_{1}[\bar{F} / \bar{X}], \ldots, \tilde{A}_{n}[\bar{F} / \bar{X}]^{5}$.

Proof. The proof is done by induction on the length of the derivation $\mathcal{D}$. See [5] pages $1306-1307$ for the $\rightarrow$-rules. ${ }^{6}$

Let us analyse the case where the last rule is a $\forall_{1} \mathrm{I}$-rule.

$$
\begin{gathered}
\Gamma[\bar{X}] \\
\vdots \\
\frac{A[\bar{X}]}{(\forall x . A)[\bar{X}]} \forall_{1} \mathrm{I}
\end{gathered}
$$

Fix $\bar{F}$ a tuple of predicate formulas. The aim is to prove that $\tilde{\Gamma}[\bar{F} / \bar{X}] \vdash_{\text {IQC }}$ $\widetilde{(\forall x \cdot A)}[\bar{F} / \bar{X}] . \quad$ According to the induction hypothesis, we have $\tilde{\Gamma}[\bar{F} / \bar{X}] \vdash$ IQC $\tilde{A}[\bar{F} / \bar{X}]$. Thus, adding a $\forall_{1}$ I-rule, we obtain $\forall x .(\tilde{A}[\bar{F} / \bar{X}])$, i.e. we obtain $(\forall x . \tilde{A})[\bar{F} / \bar{X}]$ which by Definition 2.2 is $\widetilde{\forall x . A)}[\bar{F} / \bar{X}]$. We are assuming (modulo a renaming of variables) that the bound variables in $\mathcal{D}$ do not occur free in $\bar{F}$.

Let us analyse the case where the last rule is a $\forall_{1}$ E-rule.

$$
\begin{gathered}
\Gamma[\bar{X}] \\
\vdots \\
\frac{(\forall x \cdot A)[\bar{X}]}{(A[t / x])[\bar{X}]} \forall_{1} \mathrm{E}
\end{gathered}
$$

Fix $\bar{F}$ a tuple of predicate formulas. By induction hypothesis, we have $\tilde{\Gamma}[\bar{F} / \bar{X}] \vdash_{\text {IQC }}$ $(\forall x . \tilde{A})[\bar{F} / \bar{X}]$. Applying the $\forall_{1}$ E-rule, we get $(\tilde{A}[\bar{F} / \bar{X}])[t / x] \equiv(\tilde{A}[t / x])[\bar{F} / \bar{X}]$. Again, we are assuming that bound variables in $\mathcal{D}$, in particular $x$, do not occur free in $\bar{F}$.

Let us analyse the case where the last rule is a $\forall_{2} \mathrm{I}_{-}$rule $^{7}$.

$$
\begin{gathered}
\Gamma[\bar{Y}] \\
\vdots \\
A[\bar{Y}, X] \\
\forall X . A[\bar{Y}, X]
\end{gathered}
$$

Since $\forall X . A[\bar{Y}, X]$ is a subformula of a translated formula $J^{*}$, with $J$ a predicate formula, we know that only four cases may occur: (i) $A$ is $X$; (ii) $A$ has the form $\left(D^{*} \rightarrow\left(E^{*} \rightarrow X\right)\right) \rightarrow X$; (iii) $A$ has the form $\left(D^{*} \rightarrow X\right) \rightarrow\left(\left(E^{*} \rightarrow X\right) \rightarrow X\right)$ or (iv) $A$ has the form $\forall x$. $\left(D^{*} \rightarrow X\right) \rightarrow X$, with $D$ and $E$ predicate formulas. In any

\footnotetext{
${ }^{5}$ The formula $\tilde{A}_{i}[\bar{F} / \bar{X}]$ consists in first consider the transformed formula $\tilde{A}_{i}$ and, afterwards, effect the substitution $[\bar{F} / \bar{X}]$ in it.

${ }^{6}$ In the present context, derivation in IPC is replaced by derivation in IQC and tuples $\bar{F}$ of propositional formulas are replaced by tuples of predicate formulas.

${ }^{7}$ The rules for $\forall_{2}$ are treated as in [5] (in the context of $\mathbf{F}_{\mathbf{a t}}$ ). The reason why we sketch their analysis here is because in the $\mathbf{F}_{\mathbf{a t}}^{\mathbf{Q}}$ framework we have an extra second-order universal formula to consider: the formula $\forall X .\left(\forall x .\left(D^{*} \rightarrow X\right) \rightarrow X\right)$.
} 
GILDA FERREIRA

of the cases, the only free second-order variable in $A$ is $X$. So, in the scheme above, $A[\bar{Y}, X]$ and $\forall X . A[\bar{Y}, X]$ may be replaced by $A[X]$ and $\forall X . A[X]$ respectively.

Cases (i), (ii) and (iii) follow the exact some strategy as in [5] lemma 2.2. We illustrate such strategy with case (iii).

In case (iii), the last rule is a $\forall_{2} \mathrm{I}$-rule of the form

$$
\begin{gathered}
\Gamma[\bar{Y}] \\
\vdots \\
\frac{\left(D^{*} \rightarrow X\right) \rightarrow\left(\left(E^{*} \rightarrow X\right) \rightarrow X\right)}{\forall X .\left(\left(D^{*} \rightarrow X\right) \rightarrow\left(\left(E^{*} \rightarrow X\right) \rightarrow X\right)\right)}
\end{gathered}
$$

and we need to prove that $\tilde{\Gamma}[\bar{F} / \bar{Y}] \vdash_{\text {IQC }} D \vee E$, for every tuple $\bar{F}$ of predicate formulas. Fix $\bar{F}$. By induction hypothesis, we know that $\tilde{\Gamma}[\bar{F} / \bar{Y}] \vdash$ IQC $\tilde{A}[G / X]$, for any predicate formula $G$. In particular, for $G: \equiv D \vee E$, we have

$$
\tilde{\Gamma}[\bar{F} / \bar{Y}] \vdash_{\mathbf{I Q C}}(D \rightarrow D \vee E) \rightarrow((E \rightarrow D \vee E) \rightarrow D \vee E)
$$

Thus, in the intuitionistic predicate calculus, we have the following proof

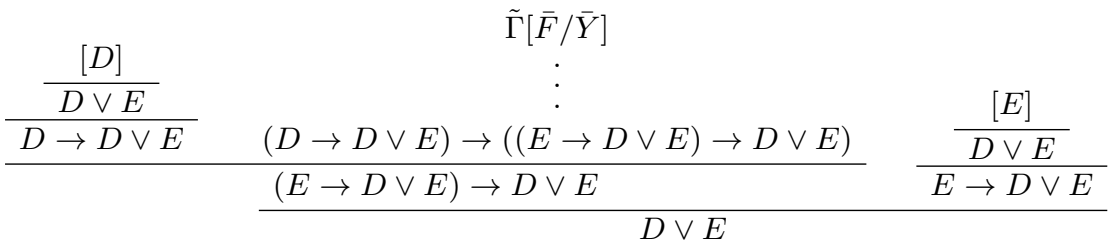

Therefore, $\tilde{\Gamma}[\bar{F} / \bar{Y}] \vdash_{\text {IQC }} D \vee E$.

In case (iv), the last rule is a $\forall_{2} \mathrm{I}$-rule of the form

$$
\begin{gathered}
\Gamma[\bar{Y}] \\
\vdots \\
\forall x .\left(D^{*} \rightarrow X\right) \rightarrow X \\
\forall X .\left(\forall x .\left(D^{*} \rightarrow X\right) \rightarrow X\right)
\end{gathered}
$$

and we need to prove that $\tilde{\Gamma}[\bar{F} / \bar{Y}] \vdash_{\text {IQC }} \exists x . D$, for every tuple $\bar{F}$ of predicate formulas. Fix $\bar{F}$. By induction hypothesis, we know that $\tilde{\Gamma}[\bar{F} / \bar{Y}] \vdash$ IQC $\forall x .(D \rightarrow$ $G) \rightarrow G$ for any predicate formula $G$. In particular, for $G: \equiv \exists x . D$, we have

$$
\tilde{\Gamma}[\bar{F} / \bar{Y}] \vdash_{\text {IQC }} \forall x .(D \rightarrow \exists x . D) \rightarrow \exists x . D .
$$

Thus, in the natural deduction calculus for the intuitionistic predicate calculus, we have the following proof

$$
\begin{array}{cc}
\frac{[D]}{\exists x . D} & \tilde{\Gamma}[\bar{F} / \bar{Y}] \\
\frac{D \rightarrow \exists x . D}{D x .(D \rightarrow \exists x . D)} & \forall x .(D \rightarrow \exists x . D) \rightarrow \exists x . D \\
\hline & \exists x . D
\end{array}
$$

Therefore, $\tilde{\Gamma}[\bar{F} / \bar{Y}] \vdash_{\text {IQC }} \exists x . D$.

Let us analyse the case where the last rule is a $\forall_{2}$ E-rule: 


$$
\begin{gathered}
\Gamma[\bar{Y}] \\
\vdots \\
\forall X . A[X, \bar{Y}] \\
\hline A[C / X, \bar{Y}]
\end{gathered}
$$

with $C$ an atomic formula in $\mathbf{F}_{\mathbf{a t}}^{\mathbf{Q}}$. We assume w.l.o.g that if $C$ is a second-order variable then $C$ is among the variables $\bar{Y}$, say $Y_{i}$.

By hypothesis, since $\forall X . A[X, \bar{Y}]$ is a subformula of a translated formula, we know that this formula falls into one of the following four cases: (i) it is the translation of $\perp$; (ii) it is the translation of a conjunction; (iii) it is the translation of a disjunction or (iv) it is the translation of a first-order existential quantification. Moreover, $\forall X . A[X, \bar{Y}]$ has no free second-order variables and so, in the scheme above we can replace $\forall X . A[X, \bar{Y}]$ and $A[C / X, \bar{Y}]$ by $\forall X . A[X]$ and $A[C / X]$, respectively.

Once again, since cases (i), (ii) and (iii) can be done mutatis mutandis as in [5] lemma 2.2 (in the context of $\mathbf{F}_{\text {at }}$ ) we just present case (iii) to illustrate how it also works in the framework of $\mathbf{F}_{\mathbf{a t}}^{\mathbf{Q}}$.

In case (iii), we have the following proof in $\mathbf{F}_{\mathbf{a t}}^{\mathbf{Q}}$

$$
\begin{gathered}
\Gamma[\bar{Y}] \\
\vdots \\
\forall X .\left(\left(D^{*} \rightarrow X\right) \rightarrow\left(\left(E^{*} \rightarrow X\right) \rightarrow X\right)\right) \\
\hline\left(D^{*} \rightarrow C\right) \rightarrow\left(\left(E^{*} \rightarrow C\right) \rightarrow C\right)
\end{gathered}
$$

with $C$ and atomic formula in $\mathbf{F}_{\text {at }}^{\mathbf{Q}}$ and we need to prove that $\tilde{\Gamma}[\bar{F} / \bar{Y}] \vdash$ IQC $(D \rightarrow$ $\left.C\left[F_{i} / Y_{i}\right]\right) \rightarrow\left(\left(E \rightarrow C\left[F_{i} / Y_{i}\right]\right) \rightarrow C\left[F_{i} / Y_{i}\right]\right)$, for any tuple $\bar{F}$ of predicate formulas. Fix $\bar{F}$. By induction hypothesis, $\tilde{\Gamma}[\bar{F} / \bar{Y}] \vdash_{\text {IQC }} D \vee E$. Thus, we have the following proof in the intuitionistic predicate calculus

$$
\begin{aligned}
& \tilde{\Gamma}[\bar{F} / \bar{Y}]
\end{aligned}
$$

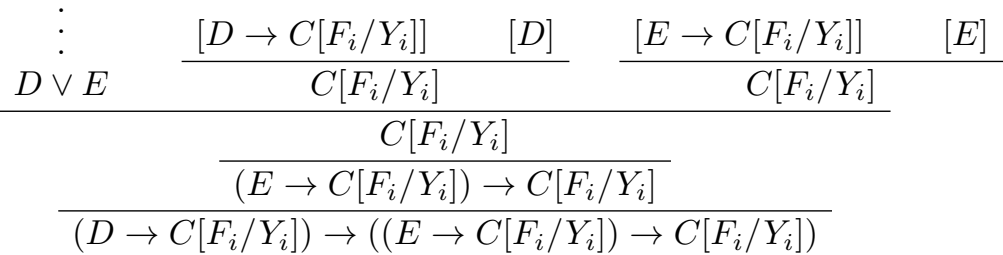

In case (iv) the last rule is a $\forall_{2}$ E-rule of the form

$$
\begin{gathered}
\Gamma[\bar{Y}] \\
\vdots \\
\frac{\forall X .\left(\forall x \cdot\left(D^{*} \rightarrow X\right) \rightarrow X\right)}{\left.\forall x .\left(D^{*} \rightarrow C\right) \rightarrow C\right)}
\end{gathered}
$$

with $C$ an atomic formula in $\mathbf{F}_{\mathbf{a t}}^{\mathbf{Q}}$, and we need to prove that $\tilde{\Gamma}[\bar{F} / \bar{Y}] \vdash_{\text {IQC }} \forall x .(D \rightarrow$ $\left.C\left[F_{i} / Y_{i}\right]\right) \rightarrow C\left[F_{i} / Y_{i}\right]$ for every tuple $\bar{F}$ of predicate formulas. Fix $\bar{F}$. By induction hypothesis, we know that $\tilde{\Gamma}[\bar{F} / \bar{Y}] \vdash$ IQC $\exists$ x.D.

Thus, we have the following proof in IQC 


$$
\begin{array}{rcc}
\frac{\left[\forall x .\left(D \rightarrow C\left[F_{i} / Y_{i}\right]\right)\right]}{D \rightarrow C\left[F_{i} / Y_{i}\right]} \quad[D] & \tilde{\Gamma}[\bar{F} / \bar{Y}] \\
\frac{C\left[F_{i} / Y_{i}\right]}{C\left[F_{i} / Y_{i}\right]} & \vdots x . D \\
\hline & \exists x .\left(D \rightarrow C\left[F_{i} / Y_{i}\right]\right) \rightarrow C\left[F_{i} / Y_{i}\right]
\end{array}
$$

Therefore, $\tilde{\Gamma}[\bar{F} / \bar{Y}] \vdash_{\text {IQC }} \forall x .\left(D \rightarrow C\left[F_{i} / Y_{i}\right]\right) \rightarrow C\left[F_{i} / Y_{i}\right]$.

Proposition 2.3 (Faithfulness). Let $\Gamma: \equiv A_{1}, \ldots, A_{n}$ and $A$ be formulas in IQC and consider their translations $\Gamma^{*}: \equiv A_{1}^{*}, \ldots, A_{n}^{*}$ and $A^{*}$ into $\mathbf{F}_{\mathbf{a t}}^{\mathbf{Q}}$.

$$
\text { If } \Gamma^{*} \vdash_{\mathbf{F}_{\mathbf{a t}}^{\mathbf{Q}}} A^{*} \text { then } \Gamma \vdash_{\mathbf{I Q C}} A \text {. }
$$

Proof. Suppose that $\Gamma^{*} \vdash_{\mathbf{F}_{\mathbf{a t}}^{\mathbf{Q}}} A^{*}$. Since $\mathbf{F}_{\mathbf{a t}}^{\mathbf{Q}}$ enjoys the normalization property (see the beginning of Section 2), we know that there is a normal proof in $\mathbf{F}_{\mathbf{a t}}^{\mathbf{Q}}$, say $\mathcal{D}$, of $A^{*}$ with premises $\Gamma^{*}$. By the subformula property (Proposition 2.1), all formulas that occur in $\mathcal{D}$ are subformulas of $A^{*}$ or are subformulas of formulas in $\Gamma^{*}$. Therefore, we are in the conditions of Lemma 2.6. Applying such lemma, we conclude that $\widetilde{\Gamma^{*}} \vdash_{\text {IQC }} \widetilde{A^{*}}$, i.e., $\Gamma \vdash_{\text {IQC }} A$.

\section{The existence property for $\mathbf{F}_{\mathrm{at}}^{\mathbf{Q}}$}

In this section we prove that $\mathbf{F}_{\mathbf{a t}}^{\mathbf{Q}}$ enjoys the existence property.

In order to simplify notation, we denote the Russell-Prawitz translation of $\exists x . A$ into $\mathbf{F}_{\text {at }}^{\mathbf{Q}}$ by $\exists x . A^{*}$. I.e., $\exists x . A^{*}$ abbreviates the formula $\forall X .\left(\forall x .\left(A^{*} \rightarrow X\right) \rightarrow X\right)$ (with $A$ a formula in IQC). Note that $\exists$ is not a primitive symbol in $\mathbf{F}_{\mathbf{a t}}^{\mathbf{Q}}$, just an abbreviation for ease of notation.

Theorem 3.1. Let $A$ be the translation into the language of $\mathbf{F}_{\mathbf{a t}}^{\mathbf{Q}}$ of a given formula of IQC.

$$
\text { If } \vdash_{\mathbf{F}_{\mathbf{a t}}^{\mathbf{Q}}} \exists x . A \text { then there exists a term } t \text { such that } \vdash_{\mathbf{F}_{\mathbf{a t}}^{\mathbf{Q}}} A[t / x] .
$$

Proof. Suppose that $\vdash_{\mathbf{F}_{\mathbf{a}}^{\mathbf{Q}}} \exists x$.A. Since $\mathbf{F}_{\mathbf{a t}}^{\mathbf{Q}}$ enjoys the (strong) normalization property (see Section 2), we can take a normal derivation $\mathcal{D}$ of $\exists x$. A, i.e. a normal proof of $\forall X .(\forall x .(A \rightarrow X) \rightarrow X)$ in $\mathbf{F}_{\mathbf{a t}}^{\mathbf{Q}}$.

The last rule of such a proof has to be an introduction rule, otherwise by Proposition 2.1 there should be an undischarged hypothesis which is not the case.

Thus we have

$$
\frac{\forall x .(A \rightarrow X) \rightarrow X}{\forall X .(\forall x .(A \rightarrow X) \rightarrow X)}
$$

Again, by Proposition 2.1, the penultimate inference is an introduction rule: 


$$
\begin{gathered}
{[\forall x \cdot(A \rightarrow X)]} \\
\frac{\dot{X}}{\forall x \cdot(A \rightarrow X) \rightarrow X} \\
\forall X .(\forall x \cdot(A \rightarrow X) \rightarrow X)
\end{gathered}
$$

Since $X$ is a second-order variable, it can not result from the application of an introduction rule, so by Proposition 2.1 we can consider the principal branch of the derivation:

$$
\begin{gathered}
\forall x .(A \rightarrow X) \\
: \\
\dot{X}
\end{gathered}
$$

More precisely, we have:

$$
\frac{\frac{\forall x .(A \rightarrow X)}{A[t / x] \rightarrow X} \quad A[t / x]}{X}
$$

for a certain term $t$.

If, in the right side of the derivation, $A[t / x]$ is derivable without (undischarged) premises, the result is immediate.

If not, necessarily, we have

$$
\begin{gathered}
\forall x .(A \rightarrow X) \\
\vdots \\
A[t / x]
\end{gathered}
$$

I.e., we have a normal proof in $\mathbf{F}_{\mathbf{a t}}^{\mathbf{Q}}$ of $A[t / x]$ from the premise $\forall x .(A \rightarrow X)$. Moreover, since $A$ is a translated formula, by Proposition 2.1, we know that any second-order universal formula in such a proof is a subformula of a translated formula, i.e. we are in the conditions of Lemma 2.5.

Applying Lemma 2.5 (with $F: \equiv X \rightarrow X)$, we have $\forall x .(A \rightarrow(X \rightarrow X)) \vdash_{\mathbf{F}_{\mathbf{a t}}^{\mathbf{Q}}}$ $A[t / x]$. Therefore we have in $\mathbf{F}_{\mathbf{a t}}^{\mathbf{Q}}$

$$
\frac{\frac{[X]}{X \rightarrow X}}{A \rightarrow(X \rightarrow X)}
$$

i.e., $\vdash_{\mathbf{F}_{\mathbf{a t}}^{\mathbf{Q}}} A[t / x]$. 


\section{The EXISTEnCE PROPERTy FOR IQC}

Illustrating the potential of the sound and faithful embedding of IQC into $\mathbf{F}_{\mathbf{a t}}^{\mathbf{Q}}$ in exporting properties of the latter calculus into the former one, we give a new proof of the existence property for the (full) intuitionistic predicate calculus. The novelty of this alternative proof is that, being a proof-theoretic proof via natural deduction, it does not rely on commuting conversions. Note that, as opposed to IQC where in order to ensure that normal proofs enjoy the subformula property commuting conversions (also known as permutative conversions) have to be added to the calculus, in $\mathbf{F}_{\text {at }}^{\mathbf{Q}}$ the connectives $\perp, \vee$ and $\exists$ are absent and there are no commuting conversions. I.e., the atomic polymorphic calculus does not suffer from the defects pointed by Girard (see Section 1) associated with the bad connectives. This explains the advantage of proving results in $\mathbf{I Q C}$ via this $\mathbf{F}_{\mathbf{a t}}^{\mathbf{Q}}$ detour. Moreover once the sound and faithful translation is in place the transference of results between the two systems, as illustrated here, becomes an immediate exercise.

Theorem 4.1. If $\vdash_{\mathbf{I Q C}} \exists x$.A then there exists a term $t$ such that $\vdash_{\mathbf{I Q C}} A[t / x]$.

Proof. Suppose that $\vdash_{\mathbf{I Q C}} \exists x A$. Since the embedding of the full intuitionistic predicate calculus into $\mathbf{F}_{\mathbf{a t}}^{\mathbf{Q}}$ is sound, we have $\vdash_{\mathbf{F}_{\mathbf{a t}}^{\mathbf{Q}}}(\exists x . A)^{*}$, i.e., $\vdash_{\mathbf{F}_{\mathrm{at}}^{\mathbf{Q}}} \exists x . A^{*}$. Applying Theorem 3.1, we know that there is $t$ such that $\vdash_{\mathbf{F}_{\mathbf{a t}}^{\mathbf{Q}}}(A[t / x])^{*}$. By the faithfulness of $\mathbf{F}_{\mathbf{a t}}^{\mathbf{Q}}$ (Proposition 2.3), we conclude that $\vdash_{\mathbf{I Q C}} A[t / x]$.

\section{ACKNOWLEDGMENTS}

The author is grateful to Fernando Ferreira for interesting and insightful discussions on predicative polymorphism.

\section{REFERENCES}

[1] Dinis, B., and G. FerReira, 'Instantiation overflow', Reports on Mathematical Logic, 51 (2016), 15-33.

[2] Ferreira, F., 'Comments on predicative logic', Journal of Philosophical Logic, 35 (2006), $1-8$.

[3] Ferreira, F., and G. Ferreira, 'Commuting conversions vs. the standard conversions of the "good" connectives', Studia Logica, 92 (2009), 63-84.

[4] Ferreira, F., and G. Ferreira, 'Atomic polymorphism', The Journal of Symbolic Logic, 78 (2013), 260-274.

[5] Ferreira, F., and G. Ferreira, 'The faithfulness of $\mathbf{F}_{\text {at }}$ : a proof-theoretic proof', Studia Logica, 103(6) (2015), 1303-1311.

[6] Ferreira, G., 'Rasiowa-Harrop disjunction property', Studia Logica, 105(3) (2017), 649-664.

[7] Ferreira, G., 'Eta-conversions of IPC implemented in atomic F', Logic Journal of the IGPL, 25(2) (2017), 115-130.

[8] Girard, J.-Y., Y. Lafont, and P. Taylor, Proofs and Types, Cambridge University Press, 1989.

[9] Prawitz, D., Natural Deduction, Almkvist \& Wiksell, Stockholm, 1965. Reprinted, with a new preface, in Dover Publications, 2006.

[10] Reynolds, J. C., 'Towards a theory of type structure', Lecture Notes in Computer Science, Colloque sur la programmation, B. Robinet, editor, Springer, 19 (1974), 408-425.

[11] Russell, B., Principles of Mathematics, George Allen and Unwin, London, 1903 (2nd edition 1937). 
Departamento de Matemática, Faculdade de Ciências da Universidade de Lisboa, Campo Grande, Ed. C6, 1749-016, Lisboa, Portugal, and Universidade Aberta, Rua Braamcamp, 90 1250-052 Lisboa, Portugal

E-mail address: gmferreira@fc.ul.pt 